\title{
Generalized Sums Based on Transcendental Functions
}

\author{
Amelia Carolina Sparavigna \\ Department of Applied Science and Technology, Politecnico di Torino, Torino, Italy
}

\begin{abstract}
In this work we are discussing the generalized sums that we can obtain from transcendental functions. The generalized sums are operations which widespread the addition of real numbers. Using these sums, we will see that we can form some Abelian groups. The study is based on the generalized sums proposed in his $\kappa$-calculus by Giorgio Kaniadakis, who used it in the framework of a generalized statistics, first applied to special relativity. Besides the investigation of some groups, the paper is also proposing examples which could be suitable for teaching purposes, in the framework of courses on theoretical physics, relativity and algebra applied to physics. Actually, the main aim of the paper is that of popularizing the existence of groups having as their operation a generalized sum.
\end{abstract}

Keywords: $\kappa$-calculus, generalized sum, groups, Abelian groups, hyperbolic functions, circular functions, logarithmic and exponential functions, theoretical physics, education.

Turin, 20 May 2018. DOI: 10.5281/zenodo.1250020

\section{Introduction}

A calculus exists, developed in the framework of a generalized statistics proposed by Giorgio Kaniadakis [1-5], which is based on deformed exponential and logarithmic functions. All the theoretical foundations and mathematical formulas of it are given in [5]. This calculus, also known as $\kappa$-calculus, has produced a series of remarkable results concerning statistics applied to many physical systems and models (see references in [5]). At the same time, it has also given new perspectives in the development economic and econometric methods [6,7].

As explained in [5], the $\kappa$-calculus turns out to be a continuous one-parameter deformation of the calculus based on the Euler exponential function. Here, we will use this calculus as a model for discussing some generalized sums based on transcendental functions. Let us note that the generalized sums are operations which widespread the addition of real numbers, and that the transcendental functions are analytic functions that do not satisfy polynomial equations, in contrast to algebraic 
functions [8]. It means that a transcendental function cannot be expressed by means of a finite sequence of algebraic operations such as addition, multiplication, and root extraction.

Using generalized sums, we can show that Abelian groups exist related to them. Besides the investigation of some groups, the paper is also proposing examples which could be suitable for teaching purposes, in the framework of courses of theoretical physics, relativity and algebra applied to physics. However, let us stress that the main aim of the paper is that of popularizing the existence of groups having as their operation a generalized sum.

\section{The א-sum}

In [5], the $\kappa$-sum is defined in the following manner. Let us consider two elements $x$ and $y$ of reals $\mathbf{R}$, and a parameter $\kappa$ real too, which is $-1<\kappa<1$. The composition law $x \oplus y$ is given by:

$$
x \oplus y=x \sqrt{1+\kappa^{2} y^{2}}+y \sqrt{1+\kappa^{2} x^{2}}
$$

which defines a generalized sum, named $\kappa$-sum. $(\mathbf{R}, \oplus)$ forms an Abelian group.

Let us remember that a group is a set $A$ having an operation - which is combining the elements of $A$. That is, the operation combines any two elements $a, b$ to form another element of the group denoted $a \bullet b$. To qualify $(A, \bullet)$ as a group, the set and operation must satisfy the following requirements. Closure: For all $a, b$ in $A$, the result of the operation $a \bullet b$ is also in A. Associativity: For all $a, b$ and $c$ in $A$, it holds $(a \bullet b) \bullet c=a \bullet(b \bullet c)$. Identity element: An element $e$ exists in $A$, such that for all elements $a$ in $A$, it is $e \bullet a=a \bullet e=a$. Inverse element: For each $a$ in $A$, there exists an element $b$ in $A$ such that $a \bullet b=$ $b \bullet a=e$, where $e$ is the identity (the notation is inherited from the multiplicative operation).

If a group is Abelian, a further requirement is the commutativity: For all $a, b$ in $A, a \bullet b=b \bullet a$. Therefore, to qualify a group as an Abelian group, the set and operation must satisfy five requirements which are known as the Abelian group axioms. A group having a not commutative operation is called a "nonabelian group" or "non-commutative group". For an Abelian group, one may choose to denote the group operation by + and the identity element by 0 (neutral element) and the inverse element as $-a$ (opposite element). In this case, the group is called an additive group.

Let us note that if a function $G(x)$ exists, which is invertible $G^{-1}(G(x))=x$, we can use it as a deformation generator [3], to generate a consequent algebra [3,9]. We will use the generator $G$ to define the group law $\Phi(x, y)$, such as in [10]:

$$
\Phi(x, y)=G\left(G^{-1}(x)+G^{-1}(y)\right)
$$


or:

$$
x \oplus y=G\left(G^{-1}(x)+G^{-1}(y)\right)
$$

In this manner the group law is giving the generalized sum of the group.

In the case of the $\kappa$-sum, the function $G$ is the hyperbolic sine:

$$
x \oplus y=\frac{1}{\kappa} \sinh (\operatorname{arsinh}(\kappa x)+\operatorname{arsinh}(\kappa y)) .
$$

In $[1,3]$, this sum is used for relativistic momenta.

\section{The generalized sum from the hyperbolic sine}

Actually, the $\kappa$-sum is a case of the generalized sum that we can obtain from the properties of the hyperbolic sine function, defined as:

$$
\operatorname{arsinh}(x)=\ln \left(x+\sqrt{1+x^{2}}\right)
$$

The domain is the whole real line. We have that [11]:

$$
\operatorname{arsinh}(x) \pm \operatorname{arsinh}(y)=\operatorname{arsinh}\left(x \sqrt{1+y^{2}} \pm y \sqrt{1+x^{2}}\right)
$$

Therefore, we have a group law:

$$
\Phi(x, y)=\sinh (\operatorname{arsinh}(x)+\operatorname{arsinh}(y))
$$

As a consequence, the sum is:

$$
x \oplus y=x \sqrt{1+y^{2}}+y \sqrt{1+x^{2}}
$$

This is the same as (1), for $\kappa=0$. The closure is given by the fact that the result of this operation is on the real line. The neutral element is 0 . The opposite element of $x$ is $-x$. Also the commutativity is evident.

To have a group, we need the discussion of the associativity too, showing that $(x \oplus y) \oplus z=x \oplus(y \oplus z)$ . Let us calculate $\operatorname{arsinh}((x \oplus y) \oplus z), \operatorname{arsinh}(x \oplus(y \oplus z))$; we can easily see that

$$
\operatorname{arsinh}((x \oplus y) \oplus z)=\operatorname{arsinh}(x \oplus y)+\operatorname{arsinh}(z)=\operatorname{arsinh}\left(x \sqrt{1+y^{2}}+y \sqrt{1+x^{2}}\right)+\operatorname{arsinh}(z),
$$


which is giving, according to (2), $\operatorname{arsinh}(x)+\operatorname{arsinh}(y)+\operatorname{arsinh}(z)$.

The same for:

$$
\operatorname{arsinh}(x \oplus(y \oplus z))=\operatorname{arsinh}(x)+\operatorname{arsinh}(y \oplus z)=\operatorname{arsinh}(x)+\operatorname{arsinh}(y)+\operatorname{arsinh}(z)
$$

\section{The generalized integers}

Let us calculate some generalized sums in the case of integer numbers. In the case of the sum of 1 and integer $n$ we have:

$$
1 \oplus n=\sqrt{1+n^{2}}+n \sqrt{2}
$$

Moreover:

$$
n \oplus n=2 n \sqrt{1+n^{2}}
$$

The generalized sum of integers is a real number.

To practice with the generalized addition $x \oplus y=x \sqrt{1+y^{2}}+y \sqrt{1+x^{2}}$, we can do the following. Let us assume to call 1 as $\alpha_{1}$ and calculate:

$$
\begin{gathered}
\alpha_{2}=\alpha_{1} \oplus \alpha_{1}=1 \oplus 1=\sqrt{2}+\sqrt{2}=2 \sqrt{2} \\
\alpha_{3}=\alpha_{1} \oplus \alpha_{2}=1 \oplus 1 \oplus 1=2 \sqrt{2} \sqrt{2}+\sqrt{1+8}=4+3=7
\end{gathered}
$$

Then we can calculate:

$$
\alpha_{2} \oplus \alpha_{2}=4 \sqrt{2} \sqrt{1+8}=12 \sqrt{2} \quad \alpha_{1} \oplus \alpha_{3}=\sqrt{50}+7 \sqrt{2}=12 \sqrt{2}
$$

So we have that:

$$
\alpha_{4}=\alpha_{2} \oplus \alpha_{2}=\alpha_{1} \oplus \alpha_{3}=1 \oplus 1 \oplus 1 \oplus 1=12 \sqrt{2}
$$

And so on. We can create a group of generalized integers $\alpha_{n}$ defined by repeating $n$ times the sum:

$$
\alpha_{n}=1 \oplus 1 \oplus 1 \ldots \oplus 1 \oplus 1
$$


The group is: $\left(\alpha_{n}, \oplus\right)$, where $n$ is a natural integer.

Now, let us assume to call the integer 2 as $\beta_{1}$ and calculate:

$$
\beta_{2}=\beta_{1} \oplus \beta_{1}=2 \oplus 2=2 \sqrt{5}+2 \sqrt{5}=4 \sqrt{5}
$$

As an exercise, we can repeat the previous calculus to obtain another group.

\section{The generalized sum from the hyperbolic tangent}

Let us consider the hyperbolic tangent. Its inverse hyperbolic function is defines as:

$$
\operatorname{artanh}(x)=\frac{1}{2} \ln \left(\frac{1+x}{1-x}\right)
$$

The domain is the open interval $(-1,1)$.

A property of this inverse function is the following [11]:

$$
\operatorname{artanh}(x) \pm \operatorname{artanh}(y)=\operatorname{artanh}\left(\frac{x \pm y}{1 \pm x y}\right)
$$

Therefore, let us define the generalized sum as:

$$
x \oplus y=\frac{x+y}{1+x y}
$$

We have a group law:

$$
\Phi(x, y)=\tanh (\operatorname{artanh}(x)+\operatorname{artanh}(y))
$$

As a consequence, we have the sum defined in (3). This sum is commutative. The neutral element is 0. The opposite element of $x$ is $-x$.

Let us discuss the associativity, showing that $(x \oplus y) \oplus z=x \oplus(y \oplus z)$. Actually:

$$
(x \oplus y) \oplus z=\frac{\frac{x+y}{1+x y}+z}{1+\left(\frac{x+y}{1+x y}\right) z}=\frac{x+y+z+x y z}{1+x y+x z+y z}
$$




$$
x \oplus(y \oplus z)=\frac{\frac{y+z}{1+y z}+x}{1+\left(\frac{y+z}{1+y z}\right) x}=\frac{y+z+x+x y z}{1+y z+y x+z x}=\frac{z+y+z+x y z}{1+x y+x z+y z}
$$

To show that we have a group $((-1,1), \oplus)$, it is necessary to verify the closure. That is, we have to see that the result of the generalized sum is in the open interval $(-1,1)$.

1

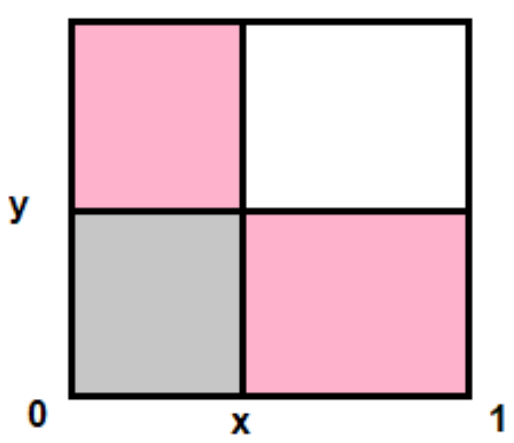

Let us assume $x>0$ and $y>0$. We need $x \oplus y<1$. And therefore:

$$
\frac{x+y}{1+x y}<1 \Rightarrow x+y<1+x y
$$

We can see that it is so, by means of a geometric approach. Let us consider a square having sides equal to 1 . In this square, let us consider rectangles $x \times 1,1 \times y$ and $x \times y$.

From the image, we can see immediately that:

$$
x+y=x \times 1+1 \times y=x y+x(1-y)+y x+y(1-x)<1+x y \Rightarrow x y+x(1-y)+y(1-x)<1
$$

The same happens in the case that $x<0$ and $y<0$. We need again $\frac{|x|+|y|}{1+|x||y|}<1$, and therefore we

1

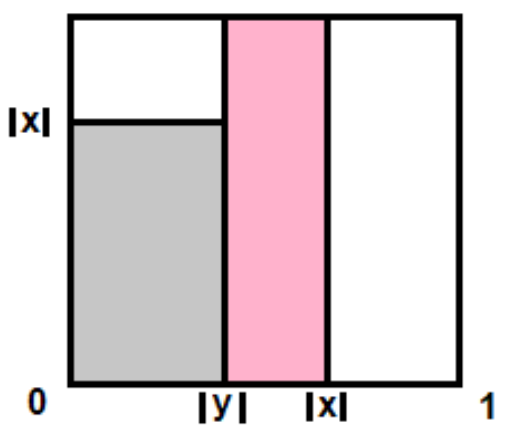
can repeat the previous approach.

In the case that $x>0$ and $y<0$, we can use the following geometry. Let us suppose $|x|>|y|$. We need to have:

$$
\frac{|x|-|y|}{1-|x||y|}<1
$$

If we look at the image on the left, we have that $|x|-|y|<1-|x||y|$, the difference $|x|-|y|$ being represented by the pink rectangle. The same is true in the other case $x<0$ and $y>0$. This means that the axiom of the closure is verified and that (($1,1), \oplus)$ is an Abelian group. 
The generalized sum (3) is used by Kaniadakis in Ref.3 for the relativistic velocity, in the following form:

$$
u_{1} \oplus u_{2}=\frac{u_{1}+u_{2}}{1+\kappa^{2} u_{1} u_{2}}
$$

where $u_{1}$ and $u_{2}$ are dimensionless velocities.

Since we are considering the group $((-1,1), \oplus)$, we avoid the divergence which we encounter when $y=-1 / x$. In fact, in relativity, the dimensionless velocity $u=v / c$ is less than 1 , if we assume $c$ as the speed of light.

\section{Another manner to generate (3)}

Let us consider another manner to generate the sum (3), using the following function and its inverse:

$$
G(x)=\frac{1-\mathrm{e}^{x}}{1+\mathrm{e}^{x}} \quad G^{-1}(x)=\ln \left(\frac{1-x}{1+x}\right)
$$

For the chosen function, we need to have $-1<x<1$. A group law $\Phi(x, y)$ could be:

$$
\Phi(x, y)=G\left(G^{-1}(x)+G^{-1}(y)\right)
$$

And therefore:

$$
\begin{gathered}
x \oplus y=G\left(G^{-1}(x)+G^{-1}(y)\right)=G\left(\ln \left(\frac{1-x}{1+x}\right)+\ln \left(\frac{1-y}{1+y}\right)\right)=G\left(\ln \left(\frac{1-x}{1+x} \frac{1-y}{1+y}\right)\right)=G(\ln Z) \\
G(\ln Z)=\frac{1-Z}{1+Z}=\frac{x+y}{1+x y}
\end{gathered}
$$

Therefore we have again the generalized sum (3):

$$
x \oplus y=\frac{x+y}{1+x y}
$$




\section{A sequence of generalized sums}

Let us consider again [10] and also [12].

As previously told, we find the group law $\Phi(x, y)$ as $\Phi(x, y)=G\left(G^{-1}(x)+G^{-1}(y)\right)$.

For the additive group law is: $\Phi(x, y)=x+y$. In this case, we can see that $G$ function is:

$$
G(z)=k z, G^{-1}(z)=k^{-1} z
$$

Then:

$$
G\left(G^{-1}(x)+G^{-1}(y)\right)=G\left(k^{-1} x+k^{-1} y\right)=k\left(k^{-1} x+k^{-1} y\right)=x+y
$$

A multiplicative group law is given by: $\Phi(x, y)=x+y+x y$.

In [10], we find that $G(z)=\mathrm{e}^{z}-1$. We can easily see that $G^{-1}(z)=\ln (z+1)$, so that:

$$
G\left(G^{-1} z\right)=\exp [\ln (z+1)]-1=z+1-1=z
$$

In this manner, we can obtain: $\quad G\left(G^{-1} x+G^{-1} y\right)=\exp [\ln (x+1)+\ln (y+1)]-1$ and

$$
\exp [\ln (x+1)+\ln (y+1)]-1=\exp [\ln ((x+1)(y+1))]-1=x+y+x y+1-1=x+y+x y
$$

The neutral element is 0 and the opposite element is:

$$
\operatorname{Opposite}(x)=-\frac{x}{1+x}
$$

However, we have to avoid $x=-1$, and not consider it in the group.

Let us note that the multiplicative group appears in the generalized sum of Tsallis entropy [13]. The related algebra has been investigated and discussed in detail in [6].

Recently, a multi-parametric version of this entropy has been proposed in [14]. This entropy is based on a rational group law:

$$
\Phi(x, y)=\frac{x+y+a x y}{1+b x y}
$$

When $b$ is equal to zero, we find the single-parametric Tsallis entropy.

In [12], we find mentioned the hyperbolic group law too: 


$$
\Phi(x, y)=\frac{x+y}{1+x y}
$$

which was discussed by Kaniadakis in [3], for the addition of velocities in special relativity. Let us stress that another hyperbolic group exists, that having (1) as generalized sum, and given in the $\kappa$ calculus as the sum of momenta [3].

In [12], we find also the Euler group law for elliptic integrals:

$$
\begin{gathered}
\Phi(x, y)=\frac{x \sqrt{1-y^{4}}+y \sqrt{1-x^{4}}}{1+x^{2} y^{2}} \\
\int_{0}^{x} \frac{d t}{\sqrt{1-t^{4}}}+\int_{0}^{y} \frac{d t}{\sqrt{1-t^{4}}}=\int_{0}^{\Phi(x, y)} \frac{d t}{\sqrt{1-t^{4}}}
\end{gathered}
$$

Let us conclude the discussion proposing two examples of generalized sums based on circular functions and another example of multiplicative group.

\section{Circular functions}

Let us discuss the generalized sums and the group laws, which are based on circular functions sine and tangent. For the circular sine, we consider the inverse circular functions, having the property [15]:

$$
\arcsin (x) \pm \arcsin (y)=\arcsin \left(x \sqrt{1-y^{2}} \pm y \sqrt{1-x^{2}}\right)
$$

The group law is: $\Phi(x, y)=\sin (\arcsin (x)+\arcsin (y))$.

Again, the generalized sum is:

$$
x \oplus y=x \sqrt{1-y^{2}}+y \sqrt{1-x^{2}}
$$

In this case we have $-1 \leqslant x, y \leqslant 1$. The group $([-1,1], \oplus)$ is Abelian.

The closure is given in the following manner.

Let us consider (4) and calculate the sine: 


$$
\sin (\arcsin (x) \pm \arcsin (y))=\sin \left(\arcsin \left(x \sqrt{1-y^{2}} \pm y \sqrt{1-x^{2}}\right)\right)
$$

This means that: $\sin (\arcsin (x) \pm \arcsin (y))=x \oplus y=x \sqrt{1-y^{2}} \pm y \sqrt{1-x^{2}}$ which is in interval $[-1,1]$. The neutral element is 0 . The opposite element of $x$ is $-x$. Also the commutativity is evident.

We have to discuss the associativity too, showing that $(x \oplus y) \oplus z=x \oplus(y \oplus z)$.

Again, let us calculate $\arcsin ((x \oplus y) \oplus z)$ and $\arcsin (x \oplus(y \oplus z))$, we can easily see:

$$
\arcsin ((x \oplus y) \oplus z)=\arcsin (x \oplus y)+\arcsin (z)=\arcsin \left(x \sqrt{1-y^{2}}+y \sqrt{1-x^{2}}\right)+\arcsin (z)
$$

which is giving, according to (4), $\arcsin (x)+\arcsin (y)+\arcsin (z)$. The same for:

$$
\arcsin (x \oplus(y \oplus z))=\arcsin (x)+\arcsin (y \oplus z)=\arcsin (x)+\arcsin (y)+\arcsin (z)
$$

In the case of the inverse circular tangent, we have the following property to use [15]:

$$
\arctan (x) \pm \arctan (y)=\arctan \left(\frac{x \pm y}{1 \mp x y}\right)
$$

Therefore, let us define the generalized sum as:

$$
x \oplus y=\frac{x+y}{1-x y}
$$

This sum is commutative. The neutral element is 0 . The opposite element of $x$ is $-x$.

For the associativity, we can show that $(x \oplus y) \oplus z=x \oplus(y \oplus z)$. Actually:

$$
\begin{gathered}
(x \oplus y) \oplus z=\frac{\frac{x+y}{1-x y}+z}{1-\left(\frac{x+y}{1-x y}\right) z}=\frac{x+y+z-x y z}{1-x y-x z-y z} \\
x \oplus(y \oplus z)=\frac{\frac{y+z}{1-y z}+x}{1-\left(\frac{y+z}{1-y z}\right) x}=\frac{y+z+x-x y z}{1-y z-y x-z x}=\frac{z+y+z-x y z}{1-x y-x z-y z}
\end{gathered}
$$


However, let us note that when we consider the sum $x \oplus y$ with $y=1 / x$, we have a divergence. This is the same as considering two angles, the sum of which being equal to 90 degrees.

\section{A multiplicative group}

Let us conclude considering the following function and its inverse:

$$
G(x)=\mathrm{e}^{-2 x}\left(\mathrm{e}^{2 x}+1\right) \quad G^{-1}(x)=\ln \left(\frac{1}{\sqrt{x-1}}\right)
$$

and investigate a possible multiplicative group from them. For the chosen function, we need to have $1<x$. A group law $\Phi(x, y)$ could be:

$$
\Phi(x, y)=G\left(G^{-1}(x)+G^{-1}(y)\right)
$$

And therefore we could imagine a generalized sum as:

$$
\begin{gathered}
x \oplus y=G\left(G^{-1}(x)+G^{-1}(y)\right)=G\left(\ln \left(\frac{1}{\sqrt{x-1}}\right)+\ln \left(\frac{1}{\sqrt{y-1}}\right)\right)=G\left(\ln \left(\frac{1}{\sqrt{x-1}} \frac{1}{\sqrt{y-1}}\right)\right)=G\left(\ln \frac{1}{Z}\right) \\
G\left(\ln \frac{1}{Z}\right)=\mathrm{e}^{-2 \ln Z}\left(e^{2 \ln Z}+1\right)=(x-1)(y-1)\left(\frac{1}{(x-1)(y-1)}+1\right) \\
x \oplus y=2-x-y+x y=(1-x)+(1-y)+x y
\end{gathered}
$$

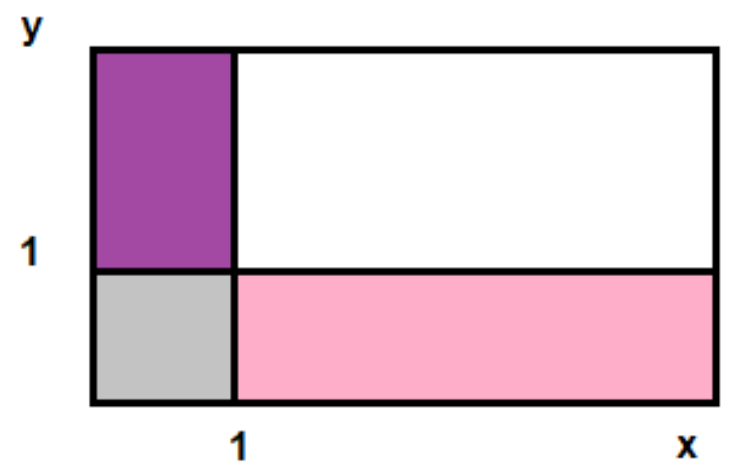

Let us consider the geometry on the left. From the rectangle $x \mathrm{x} y$, we can remove the colored rectangles $(x-1) \times 1,(y-1) \times 1$; the result is greater than 1 . So it seems that have the closure.

Now, we need to consider the neutral and opposite elements.

As we can see from (6), the neutral element is not 0 . In fact:

$$
x \oplus 0=2-x-0+x 0=(1-x)+(1-0)+x 0=2-x \neq x
$$


Let us use as a neutral element the integer 2. $x \oplus 2=(1-x)+(1-2)+2 x=1-x-1+2 x=x$.

The opposite element of $x$ is defined by $\quad x \oplus$ Opposite $(x)=2$. We have:

$$
\text { Opposite }(x)=\frac{x}{x-1}
$$

In this case, the opposite element is greater than 1 and then it is an element of the group.

Therefore, we consider 2 as the neutral element, and the opposite element as given by (8).

To have a group, we need to have the associativity $(x \oplus y) \oplus z=x \oplus(y \oplus z)$ for the given sum :

$$
x \oplus y=2-x-y+x y=(1-x)+(1-y)+x y
$$

Let us evaluate:

$$
\begin{gathered}
(x \oplus y) \oplus z=2-(x \oplus y)-z+(x \oplus y) z=2-2+x+y-x y-z+2 z-x z-y z+x y z \\
(x \oplus y) \oplus z=x+y+z-x y-x z-y z+x y z
\end{gathered}
$$

And:

$$
\begin{gathered}
x \oplus(y \oplus z)=2-x-(y \oplus z)+x(y \oplus z)=2-x-(2-y-z+y z)+x(2-y-z+y z) \\
x \oplus(y \oplus z)=x+y+z-x y-x z-y z+x y z
\end{gathered}
$$

From (8) and (9), we have the associativity. The commutativity is evident.

As a conclusion, the group having elements in the set $x>1$, for the generator $G(x)=\mathrm{e}^{-2 x}\left(\mathrm{e}^{2 x}+1\right)$, has the generalized sum given by $\quad x \oplus y=2-x-y+x y=(1-x)+(1-y)+x y$. The neutral element is 2 and the opposite element of $x$ is $\quad x /(x-1)$.

To conclude, let us note that the same approach can be used for many other transcendental functions, such as for algebraic functions.

Acknowledgment The author is indebted to Giorgio Kaniadakis and Antonio Scarfone for the valuable discussions on $\kappa$-calculus and related relativistic and kinetic problems.

\section{References}

1. Kaniadakis, G. (2001). Non-linear kinetics underlying generalized statistics. Physica A, 296, 405425. 
2. Kaniadakis, G. (2002). Statistical mechanics in the context of special relativity. Phys. Rev. E, 66, 056125 .

3. Kaniadakis, G. (2005). Statistical mechanics in the context of special relativity II. Phys. Rev. E, 72, 036108

4. Kaniadakis, G., Lissia, M., \& Scarfone, A. M. (2005). Two-parameter deformations of logarithm, exponential, and entropy: A consistent framework for generalized statistical mechanics. Physical Review E, 71(4), 046128.

5. Kaniadakis, G. (2013). Theoretical Foundations and Mathematical Formalism of the Power-Law Tailed Statistical Distributions. Entropy, 15, 3983-4010.

6. Clementi, F., Gallegati, M., \& Kaniadakis, G. (2012). A New Model of Income Distribution: The KGeneralized Distribution. Journal of Economics, Vol. 105, No. 1, 2012. Available at SSRN: https://ssrn.com/abstract=1287099 DOI: 10.2139/ssrn.1287099

7. Clementi, F., Gallegati, M., \& Kaniadakis, G. (2012). A Generalized Statistical Model for the Size Distribution of Wealth. J. Stat. Mech. (2012) P12006. Available at SSRN: https://ssrn.com/abstract=2150132 DOI: 10.2139/ssrn.2150132

8. http://mathworld.wolfram.com/TranscendentalFunction.html

9. Scarfone, A. M. (2013). Entropic forms and related algebras. Entropy, 15(2), 624-649.

10. Sicuro, G., \& Tempesta, P. (2016). Groups, information theory, and Einstein's likelihood principle. Phys. Rev. E 93, 040101(R).

11. Abramowitz, M., \& Stegun, I. (1964). Handbook of Mathematical Functions with Formulas, Graphs, and Mathematical Tables. http://people.math.sfu.ca/ cbm/aands/page_87.htm

12. Tempesta, P. (2015). Groups, generalized entropies and L-series. Templeton Workshop on Foundations of Complexity, October 2015. http://www.cbpf.br/ complex/Files/talk_tempesta.pdf 13. Tsallis, C. (1988). Possible generalization of Boltzmann-Gibbs statistics. Journal of statistical physics, 52(1-2), 479-487.

14. Curado, E. M., Tempesta, P., \& Tsallis, C. (2016). A new entropy based on a group-theoretical structure. Annals of Physics, 366, 22-31.

15. Abramowitz, M., \& Stegun, I. (1964). Handbook of Mathematical Functions with Formulas, Graphs, and Mathematical Tables. http://people.math.sfu.ca/ cbm/aands/page_80.htm 